Supporting Information

\title{
Flower- and Grass-like Self-Assemblies of an Oleanane-Type \\ Triterpenoid Erythrodiol: Application in the Removal of Toxic Dye from Water
}

Saikat Kumar Panja and Braja Gopal Bag*

Department of Chemistry \& Chemical Technology

Vidyasagar University Midnapore 721102 West Bengal, India

Fax: 00913222275297 Email: brajagb@gmail.com 
PCM model version 9.2 and DFT calculation

2. Erthrodiol from oleanolic acid

3. 1H NMR, 13C NMR and FTIR

5. Methods of sample preparation

6. Electron Microscopy images 


\section{Energy minimized structure of erythrodiol 1:}

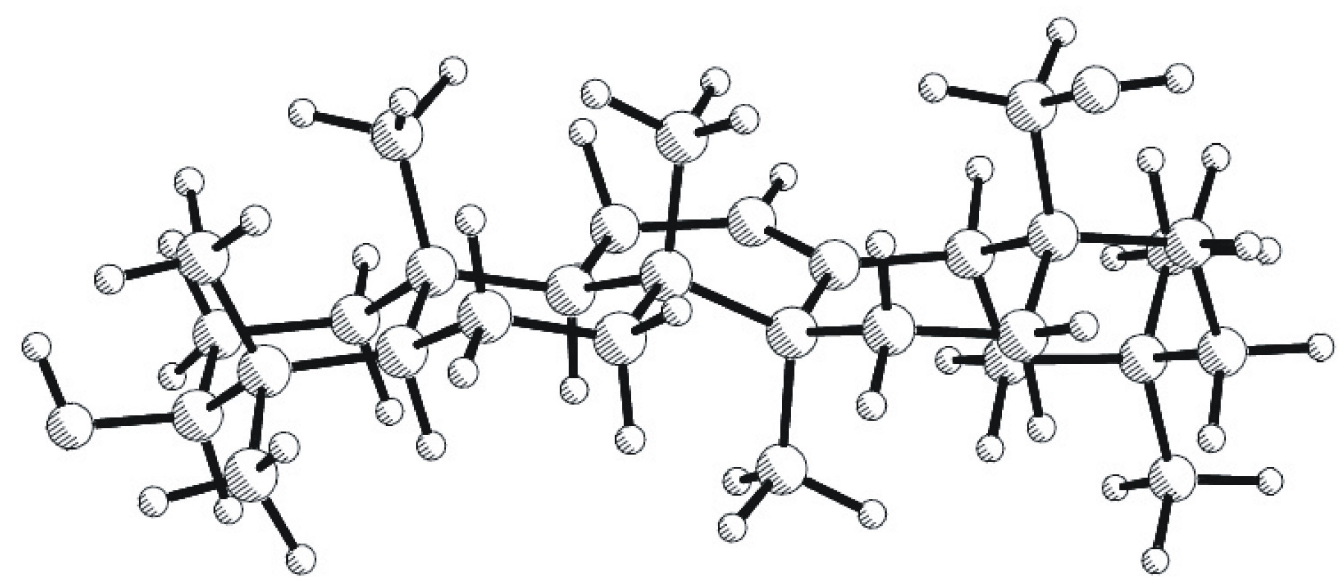

\section{$1.57 \mathrm{~nm}$}

Figure S1: Energy minimized structure of Erythrodiol 1 using MMX force field as implemented in PCMODEL version 9.2 (serena software) ${ }^{\circledR}$.

\section{Energy minimized structure of erythrodiol 1 using DFT calculation:}

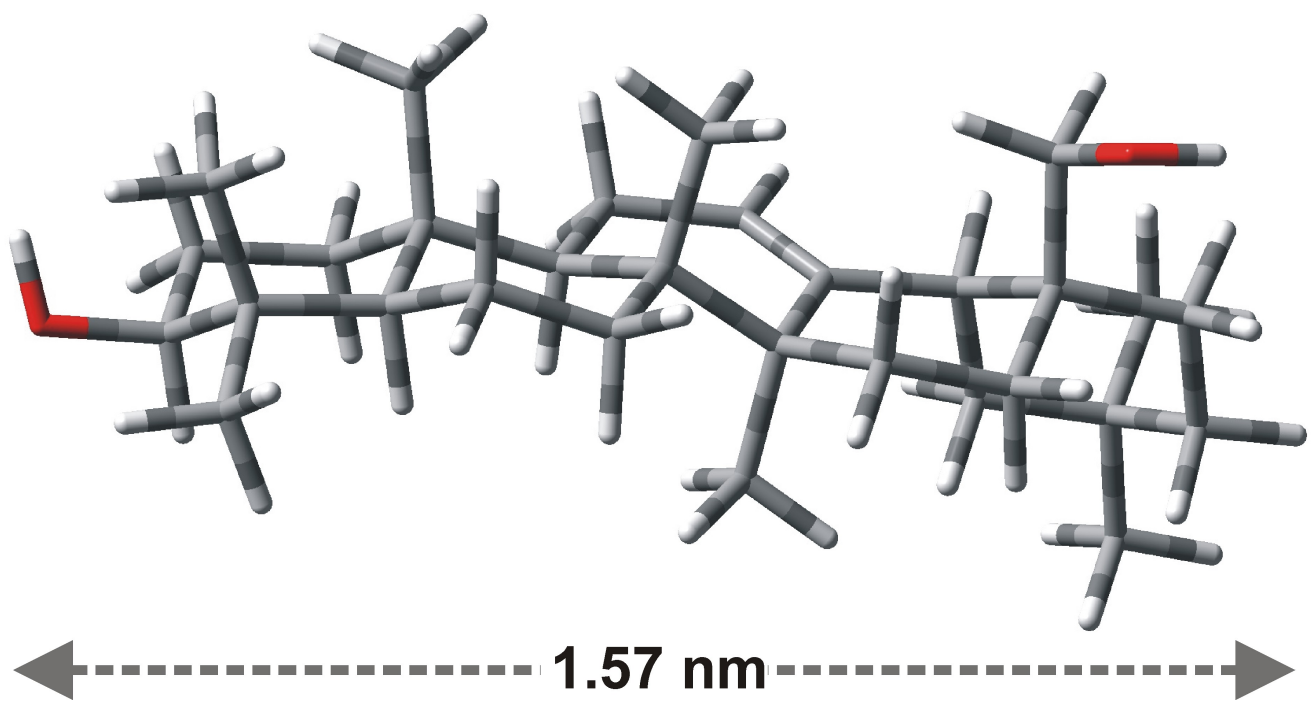

Figure S2: Energy minimized structure of Erythrodiol 1 obtained from DFT using (Gaussian 09 software) ${ }^{\circledR}$. 


\section{Reduction of oleanolic acid to yield erythrodiol:}

Erythrodiol was synthesized by one-step reduction of oleanolic acid using Lithium aluminum hydride. Oleanolic acid $(0.2 \mathrm{~g}, 0.4 \mathrm{mM})$ was taken in a $10 \mathrm{~mL}$ round bottom flask and dissolved in dry distilled THF $(1 \mathrm{~mL})$. Then $\mathrm{LiAlH}_{4}(0.08 \mathrm{~g}, 2.2 \mathrm{mM})$ was added to the solution. The reaction mixture become a lump. Then dry distilled THF $(2 \mathrm{~mL})$ was added again and the reaction mixture was stirred overnight. After that the reaction mixture was diluted with ethyl acetate $(1 \mathrm{~mL})$ and acidified with dilute $\mathrm{HCl} 1(\mathrm{~N})$. The reaction mixture was diluted with ethyl acetate $(30 \mathrm{~mL})$ and washed with brine $(10 \mathrm{~mL} \mathrm{X} \mathrm{3)}$ and dried over anhydrous sodium sulphate. Then the volatiles were removed under reduced pressure. The crude product thus obtained was purified by column chromatography (Si-gel, 100-200 mesh, $1.2 \times 10 \mathrm{~cm}$ ) using $1-20 \%$ ethyl acetate/ petroleum ether as the eluent. The product appeared as a white solid $(0.12 \mathrm{~g}$, yield $=$ $60 \%)$.

\section{Spectral Data}

${ }^{1} \mathrm{H}$ NMR (400 MHz, $\left.\mathrm{CDCl}_{3}\right): \delta 5.21(\mathrm{t}, J=3.6 \mathrm{~Hz}, \mathrm{H}-12) ; 3.59$ (d, $\left.J=11.2 \mathrm{~Hz}, \mathrm{H}-28 \mathrm{a}\right), 3.24$ (d, $J=10.8 \mathrm{~Hz}, \mathrm{H} 28 \mathrm{~b}$ ), 3.22 (m, H-3), 1.18 (s, Me-30), 1.01 (s, Me-27), 0.95 (s, Me-29), 0.94 (s, Me23), 0.90 (s, Me-26), 0.88 (s, Me-25), 0.80 (s, Me-24).

${ }^{13} \mathrm{C}$ NMR $\left(100 \mathrm{MHz}, \mathrm{CDCl}_{3}\right) \delta: 144.2,122.3,79.01,69.7,55.15,47.56,46.45,42.33,41.71$, $39.77,38.78,38.58,36.93,34.08,33.21,32.56,31.04,30.96,28.09,27.20,25.95,25.54$, $23.59,23.53,21.98,18.35,16.7,15.59,15.52$

ATR-FTIR (v, cm $\left.{ }^{-1}\right): 3357$ (w), 2933 (s), 2864 (s), 1690 (s), 1461 (s), 1387 (s), 1260 (s), 1090 (s), $1020(\mathrm{~s}), 795$ 


\section{4. ${ }^{1} \mathrm{H}$ NMR of Erythrodiol (400 MHz, CDCl3)}

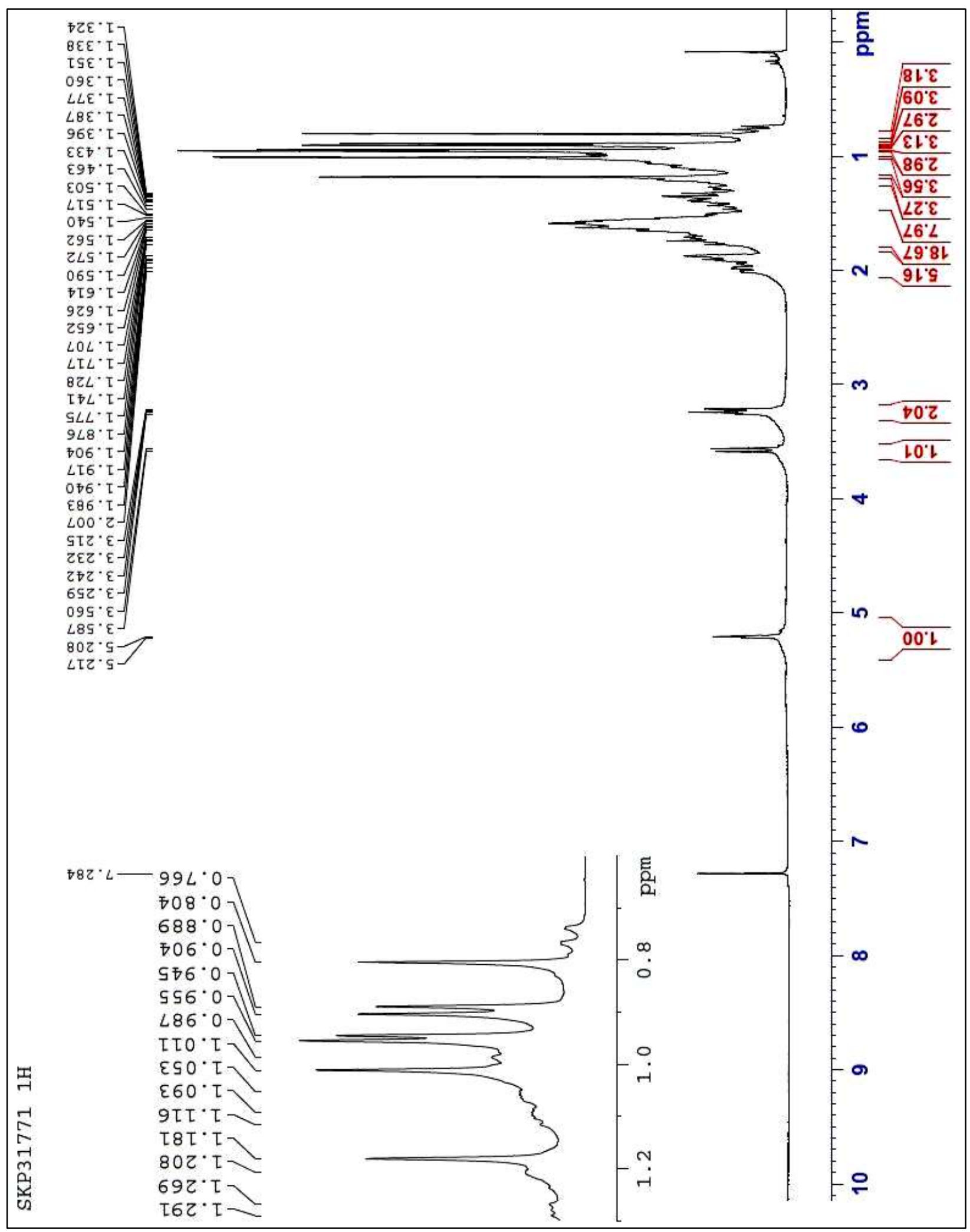

Figure S3: $1 \mathrm{H}$ NMR of Erythrodiol in $\mathrm{CDCl}_{3}$ 


\section{5. ${ }^{13} \mathrm{C}$ NMR of Erythrodiol (100 MHz, CDCl3)}

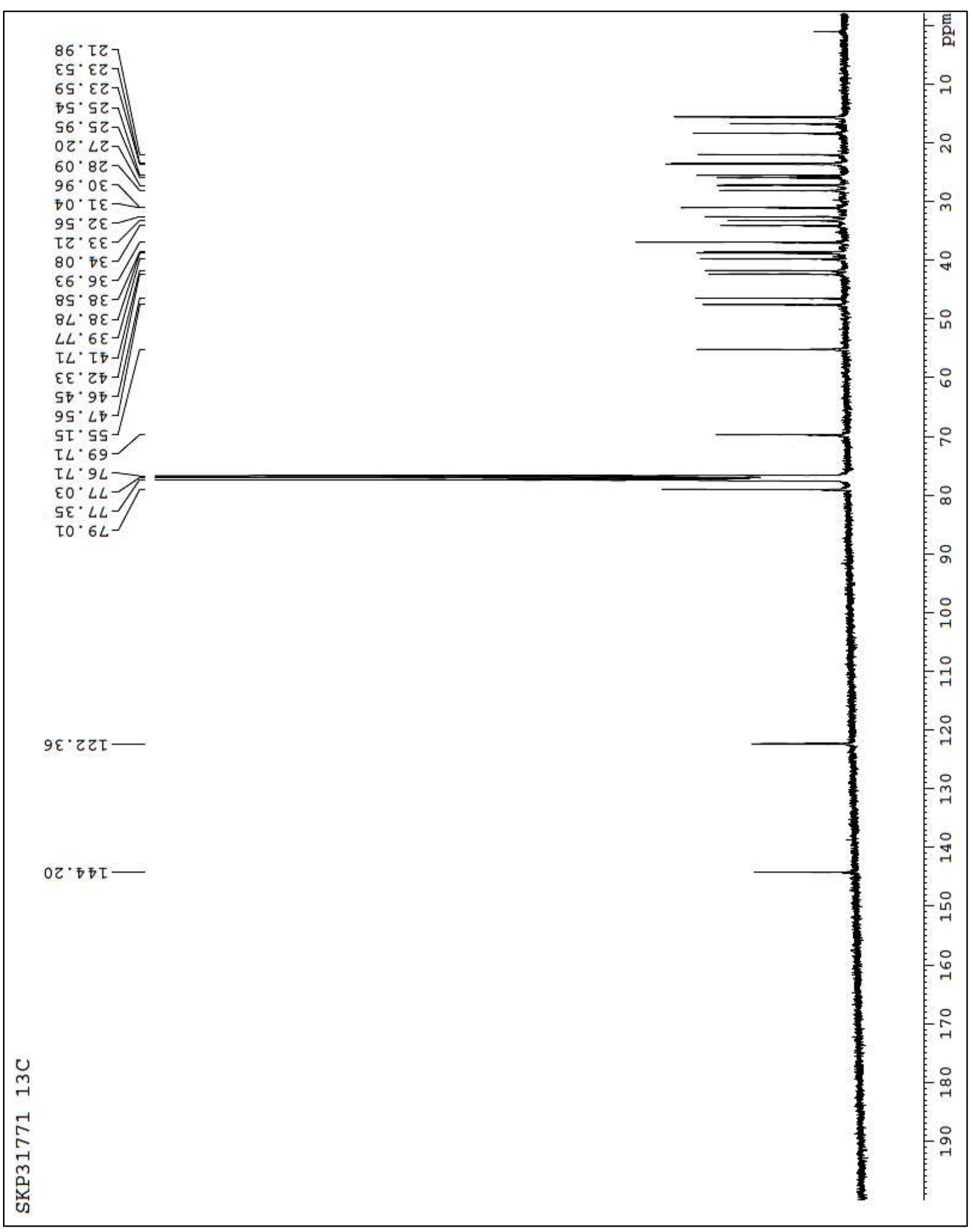

Figure S4: 13C NMR of Erythrodiol in $\mathrm{CDCl}_{3} \quad \mathrm{~S} 6$ 
6. FTIR spectra of Erythrodiol (100 MHz, CDCl3)

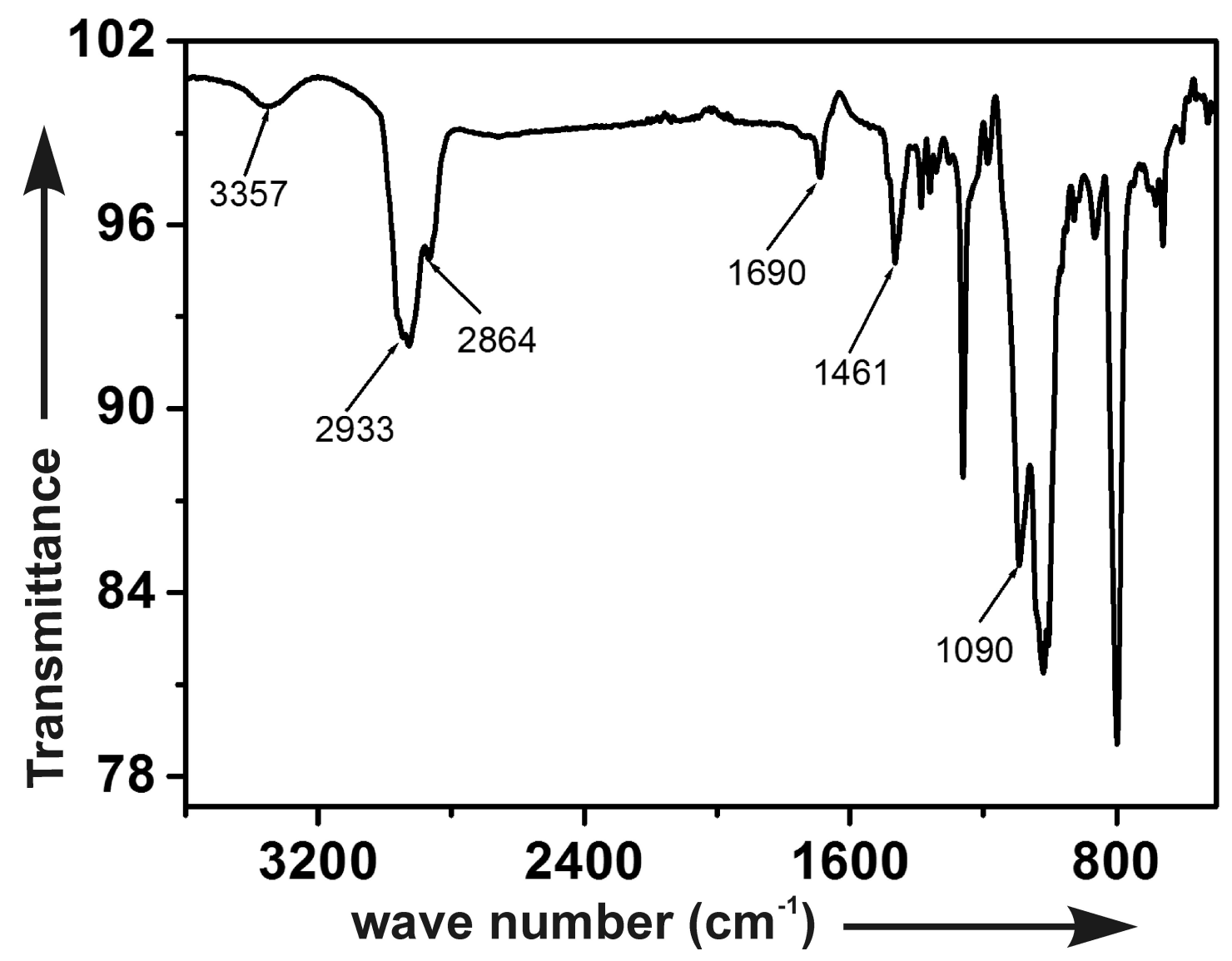

Figure S5: FTIR of Erythrodiol (neat) 


\section{Study of self-assembly properties}

For studying self-assembly behavior of erythrodiol 1, a weighed amount of 1 (2-5 mg) was taken in a clean and dried vial (capacity $=4 \mathrm{~mL}, 1 \mathrm{~cm}$ id) and was dissolved in organic liquids (usually $100-200 \mu \mathrm{L}$ ) by heating with magnetic stirring to obtain a clear solution. The vial containing the solution of $\mathbf{1}$ was then sealed tightly and allowed to cool down at room temperature and observed visually after 4-8 h. For the study of selfassembly properties in aqueous binary liquid mixtures, the hot clear solution of $\mathbf{1}$ was treated with distilled water $(20-100 \mu \mathrm{L})$ and then heated along with magnetic stirring over hot plate to obtain a clear solution. Then the resulting mixture was kept at room temperature and observed after 4-8 h.

\section{Method of sample preparation and characterization}

SEM and FESEM Samples were prepared by placing a dilute colloidal suspension of the sample on an aluminum plate and then allowed to dry initially in air for $24 \mathrm{~h}$ and then under reduced pressure for $12 \mathrm{~h}$. The samples were sputter coated with Au for 165-180 s before using and analysed using a Zeiss Scanning Electron Microscope (EVO 18). For optical microscopy (OM), an aliquot of sample was placed on a glass plate and covered with a cover slip and examined using a Nikon LV100 POL microscope with D FL Epi-fluorescence attachment. FTIR spectra of the neat powder and dried self-assemblies were analyzed by using a Perkin Elmer Spectrum Two model with $\mathrm{KBr}$ pellet. NMR spectra were recorded in a Bruker $400 \mathrm{MHz} \mathrm{NMR}$ and mass spectral analysis was carried out using Shimadzu Q2010 plus model. For wide angle X-ray diffraction studies, a dried self-assemblies of 1 were prepared from colloidal suspension of 1 and was taken on a glass plate and the volatiles were removed initially in air and then under reduced 
pressure for $12 \mathrm{~h}$ and the diffractions were recorded in a Bruker X-ray diffractometer at $25^{\circ} \mathrm{C}$ using $\mathrm{Cu}-\mathrm{K} \alpha$ filament $(\lambda=1.789 \AA)$.

\section{Electron microscopy images of the self-assemblies of 1:}
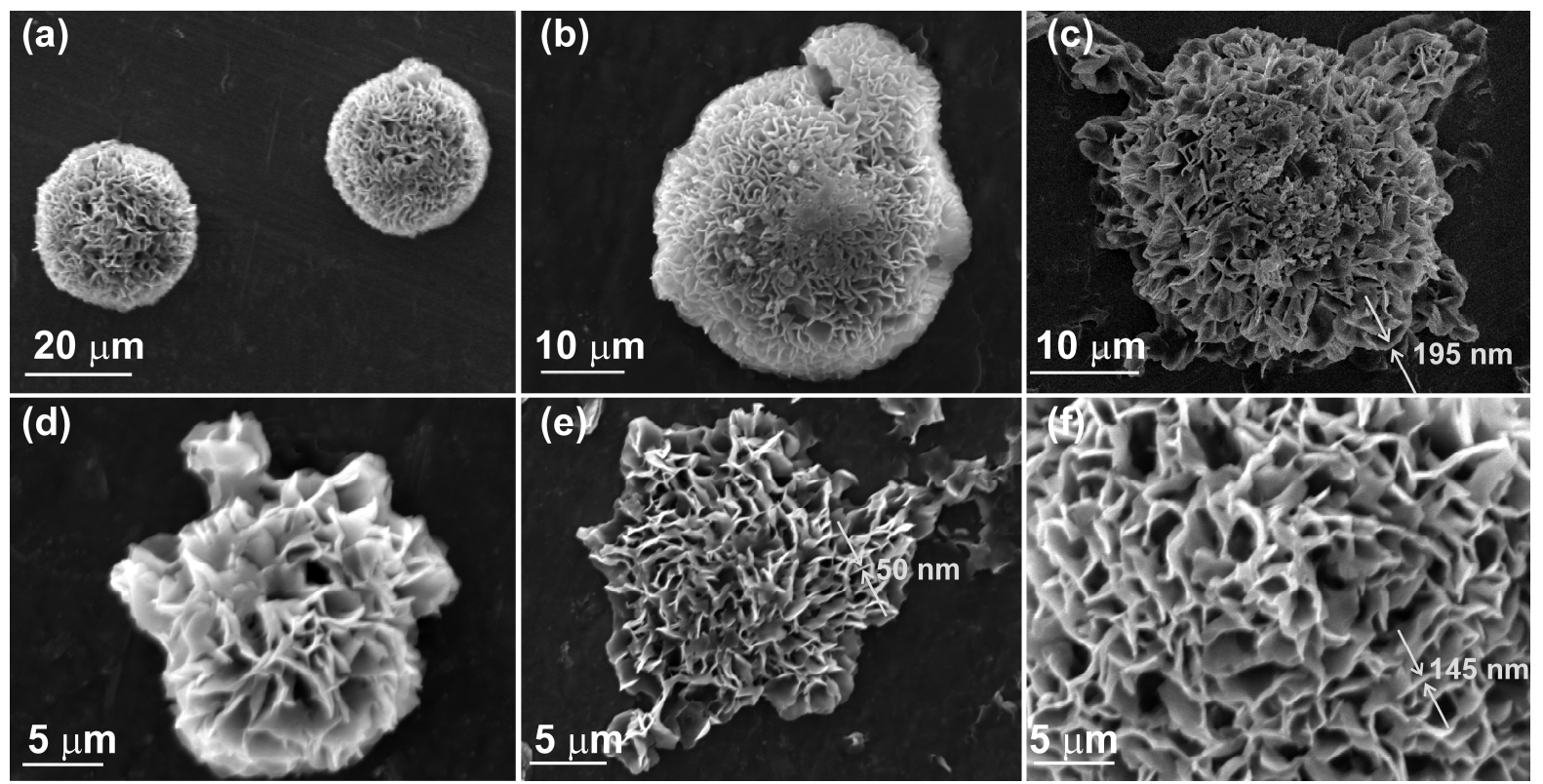

$5 \mu \mathrm{m}$

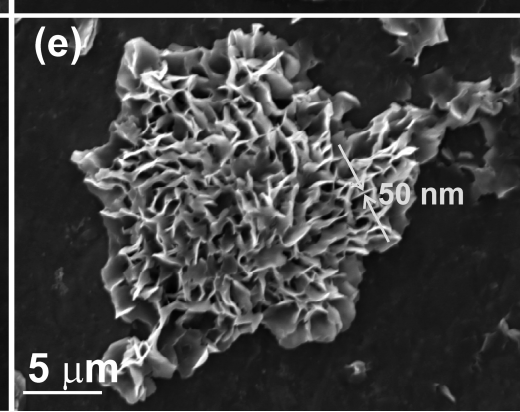

Figure S6: Electron microscopy (EM) images of dried self-assemblies of 1 prepared in oxylene $(\mathrm{a} . \mathrm{b}, \mathrm{c})(2 \% \mathrm{w} / \mathrm{v}),(\mathrm{d}, \mathrm{e}, \mathrm{f})(2.5 \% \mathrm{w} / \mathrm{v})$ 


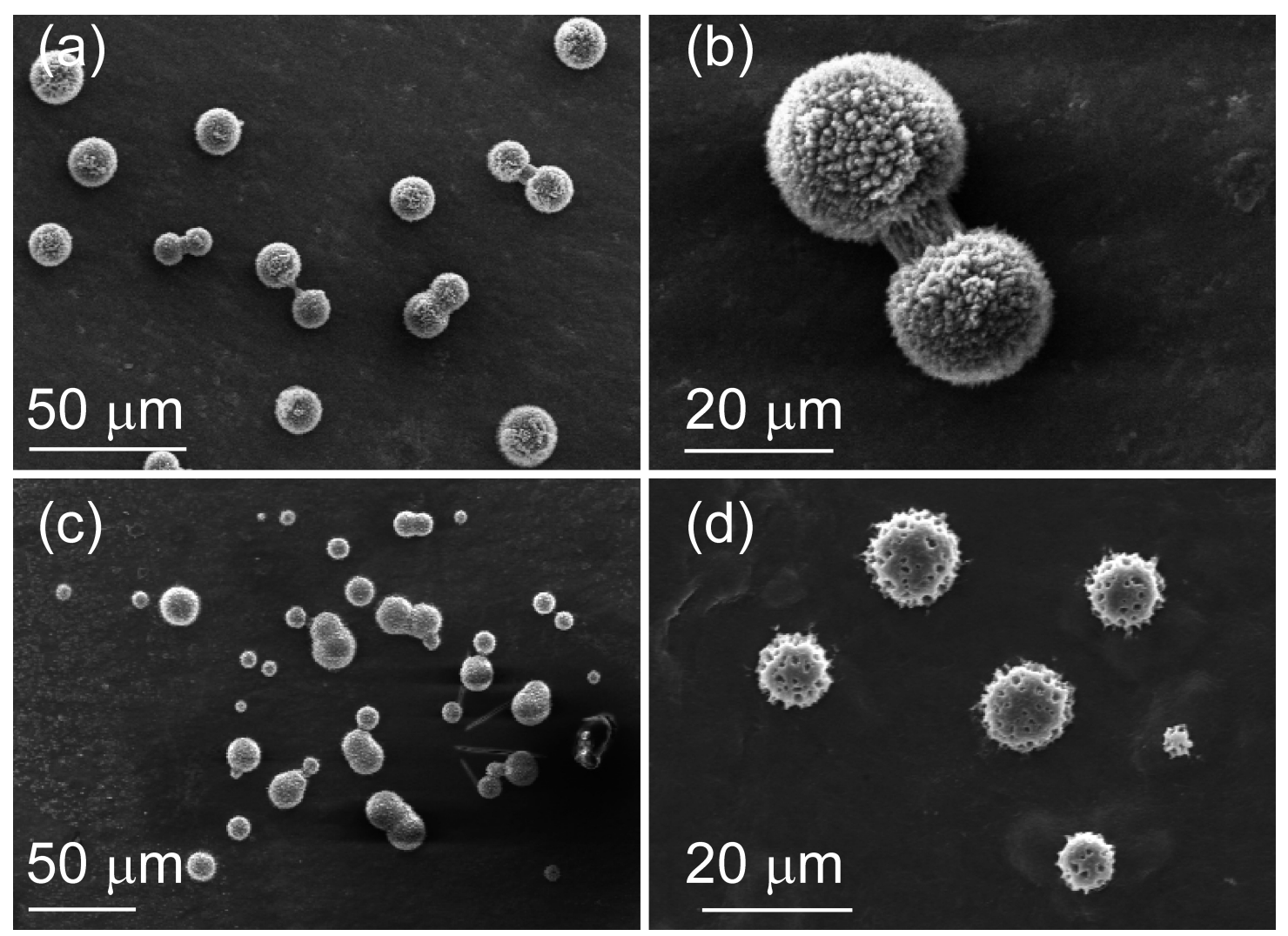

Figure S7: SEM images of dried self-assemblies of 1 prepared from $(a, b) \quad m-x y l e n e$ $(2 \% \mathrm{w} / \mathrm{v}),(\mathrm{c}, \mathrm{d})$ in $\mathrm{p}$-xylene $(2.0 \% \mathrm{w} / \mathrm{v})$
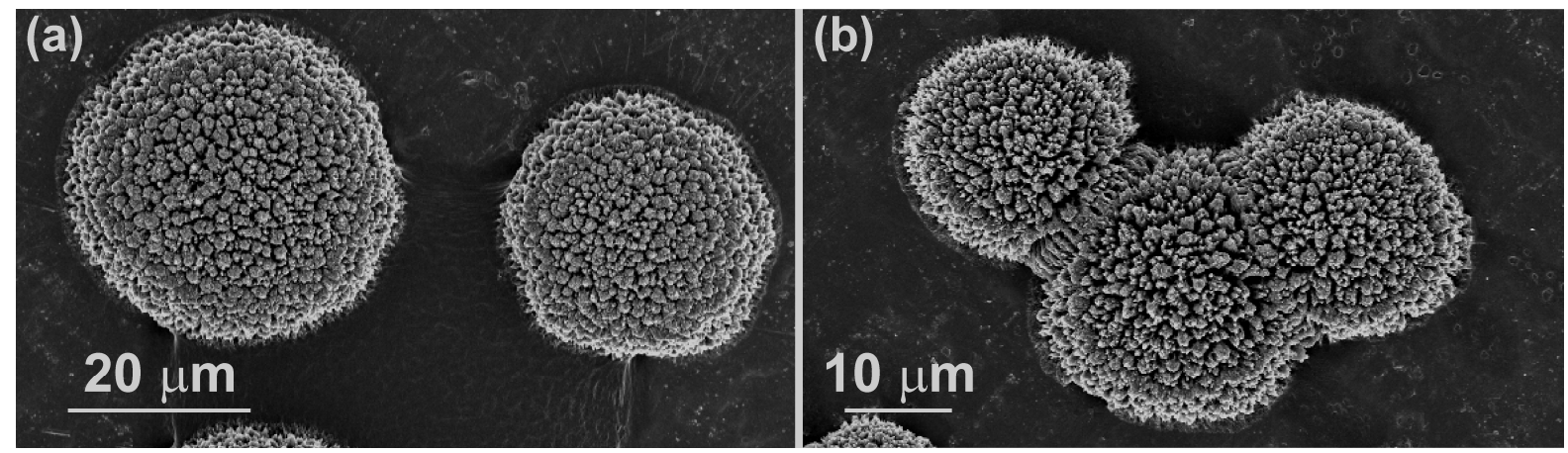

Figure S8: $(a, b)$ FESEM images of dried self-assemblies of 1 prepared from mesitylene $(2.0 \% \mathrm{w} / \mathrm{v})$ 


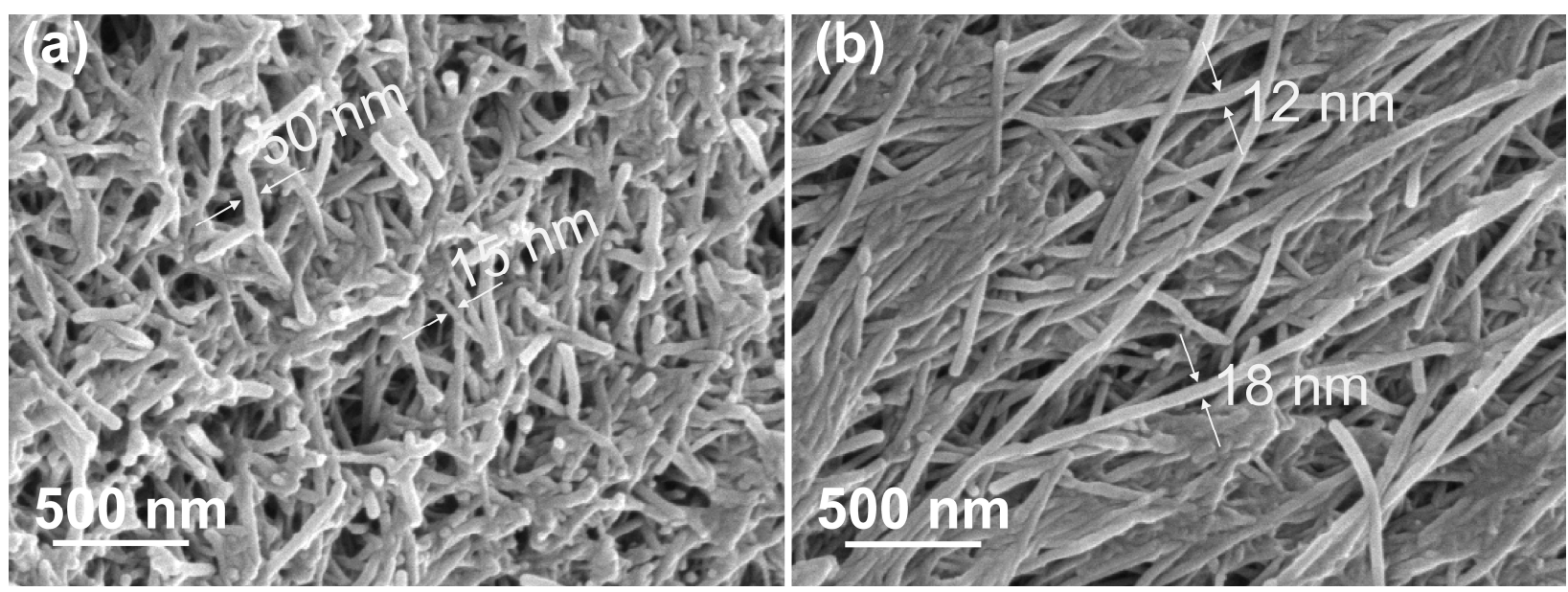

Figure S9: FESEM images of dried self-assemblies of erythrodiol prepared from (a) $\mathrm{Ph}-\mathrm{cl}(2 \%$ $\mathrm{w} / \mathrm{v}),(\mathrm{b})$ in o-dichlorobenzene $(1.5 \% \mathrm{w} / \mathrm{v})$.

\section{Histogram of the self-assemblies of 1:}
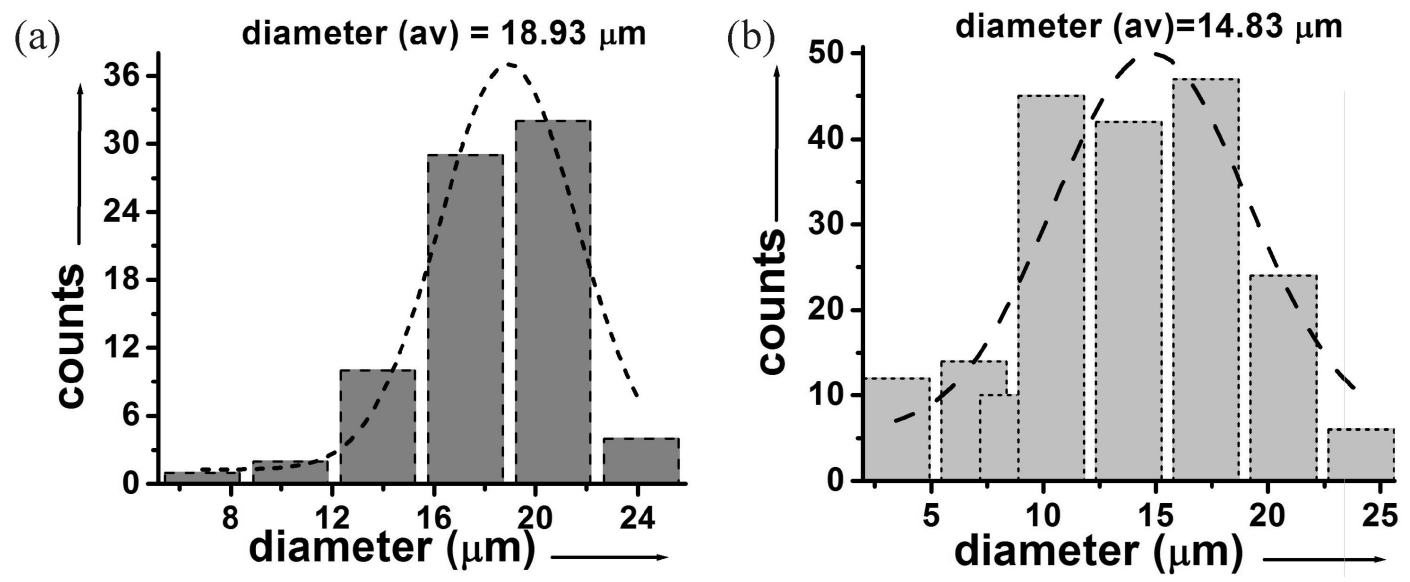

Figure S10: Histogram of spherical shaped flower (a) mesitylene ( $2 \% \mathrm{w} / \mathrm{v}),(\mathrm{b})$ m-xylene $(2 \% \mathrm{w} / \mathrm{v})$ 


\section{HRTEM of the self-assemblies of 1 :}
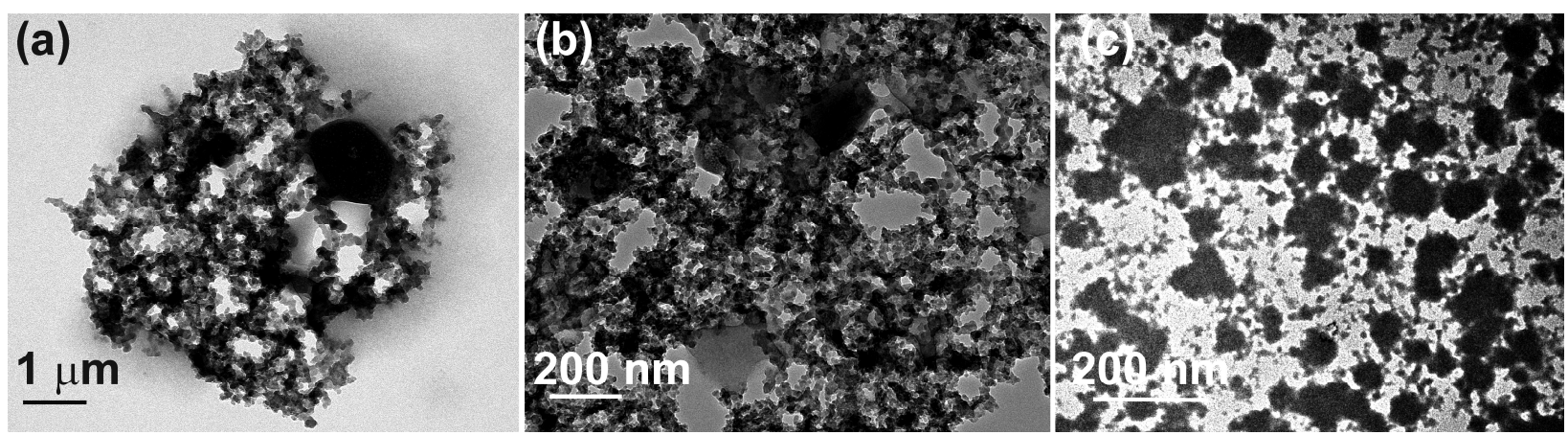

Figure S11: TEM images of dried self-assemblies of $1(\mathrm{a}, \mathrm{b})$ o-xylene $(0.7 \% \mathrm{w} / \mathrm{v}),(\mathrm{c})$ mesitylene $(0.8 \% \mathrm{w} / \mathrm{v})$.

\section{AFM of the self-assemblies of 1:}
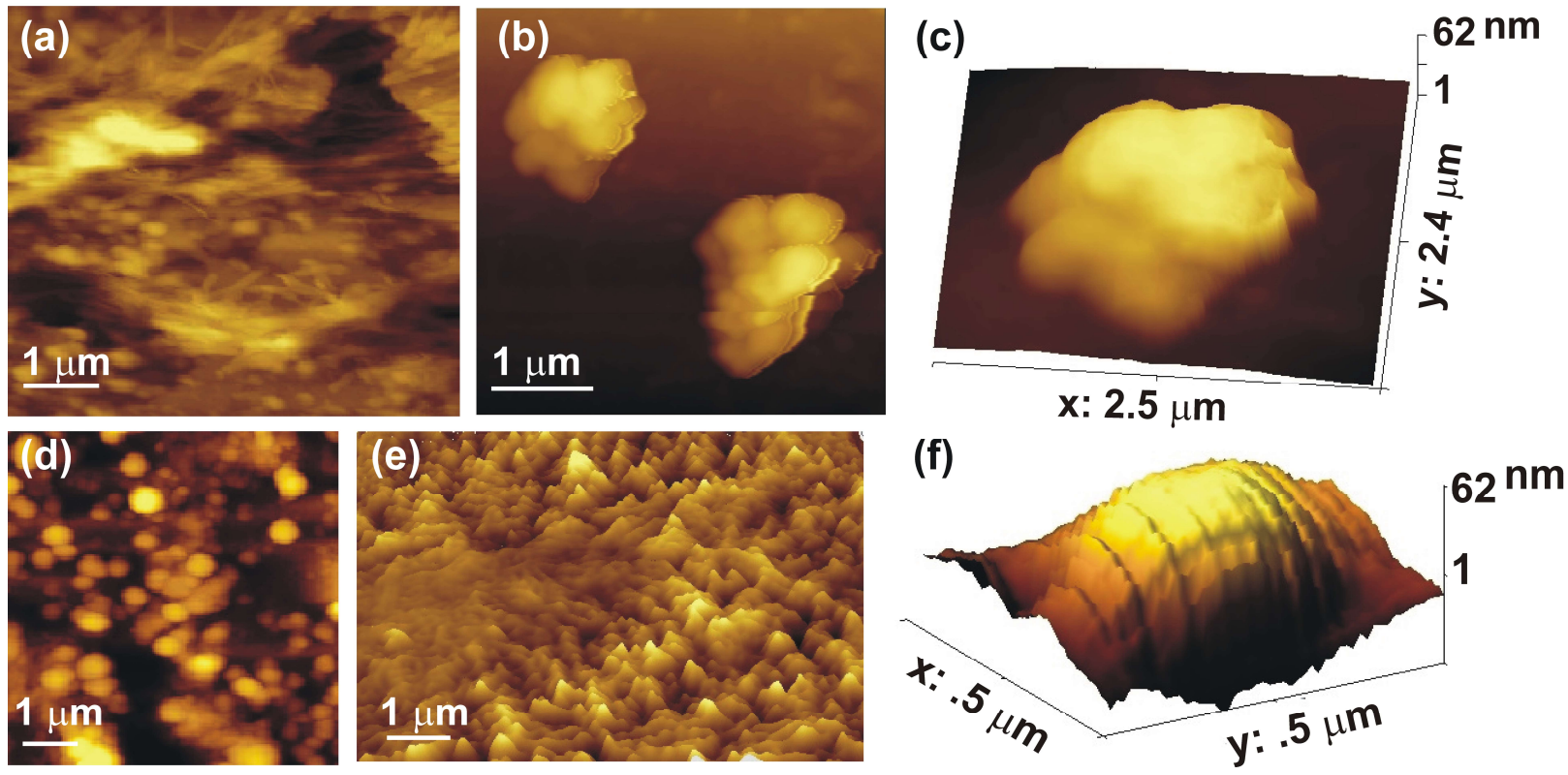

Figure S12: AFM images of self-assembled 1: (a-c) in p-xylene $(0.8 \% \mathrm{w} / \mathrm{v}),(\mathrm{d}-\mathrm{f})$ in mesitylene $(0.8 \% \mathrm{w} / \mathrm{v})$ 


\section{OM of the self-assemblies of 1 :}
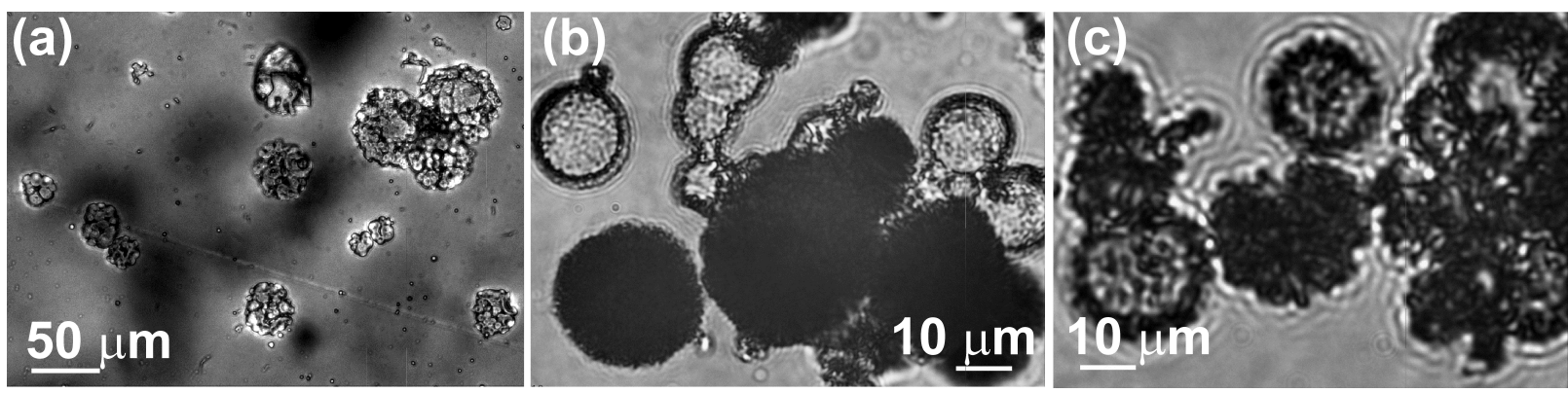

Figure S13: OPM images of 1 (a) in 2-propanol (2\% w/v), (b) in mesitylene $(2 \% \mathrm{w} / \mathrm{v}),(\mathrm{c})$ o-xylene $(2 \% \mathrm{w} / \mathrm{v})$ in native state

\section{Epifluoroscence microscopy of the self-assemblies of 1:}

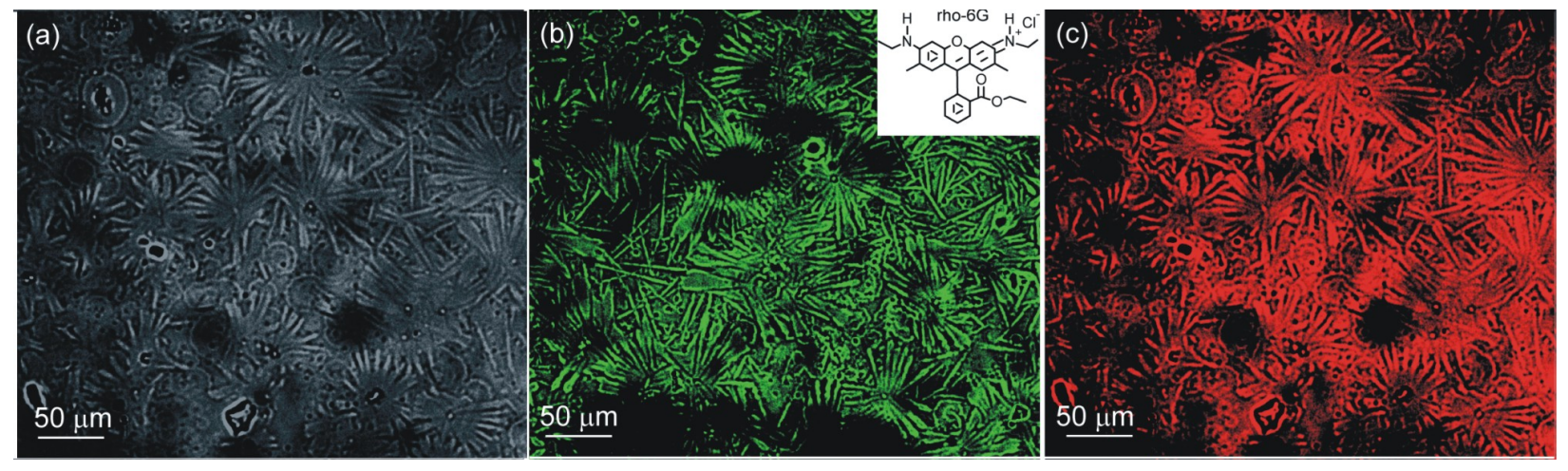

Figure S14: Epifluorescence microscopy images of adsorbed Rhodamine-6G on the porous fibrillar surface of 1 in 2-propanol $(2 \% \mathrm{w} / \mathrm{v})$ : (a) under normal light, (b) under blue light, (c) under green light. 


\section{FTIR spectrum of the self-assemblies of $1: 33$}

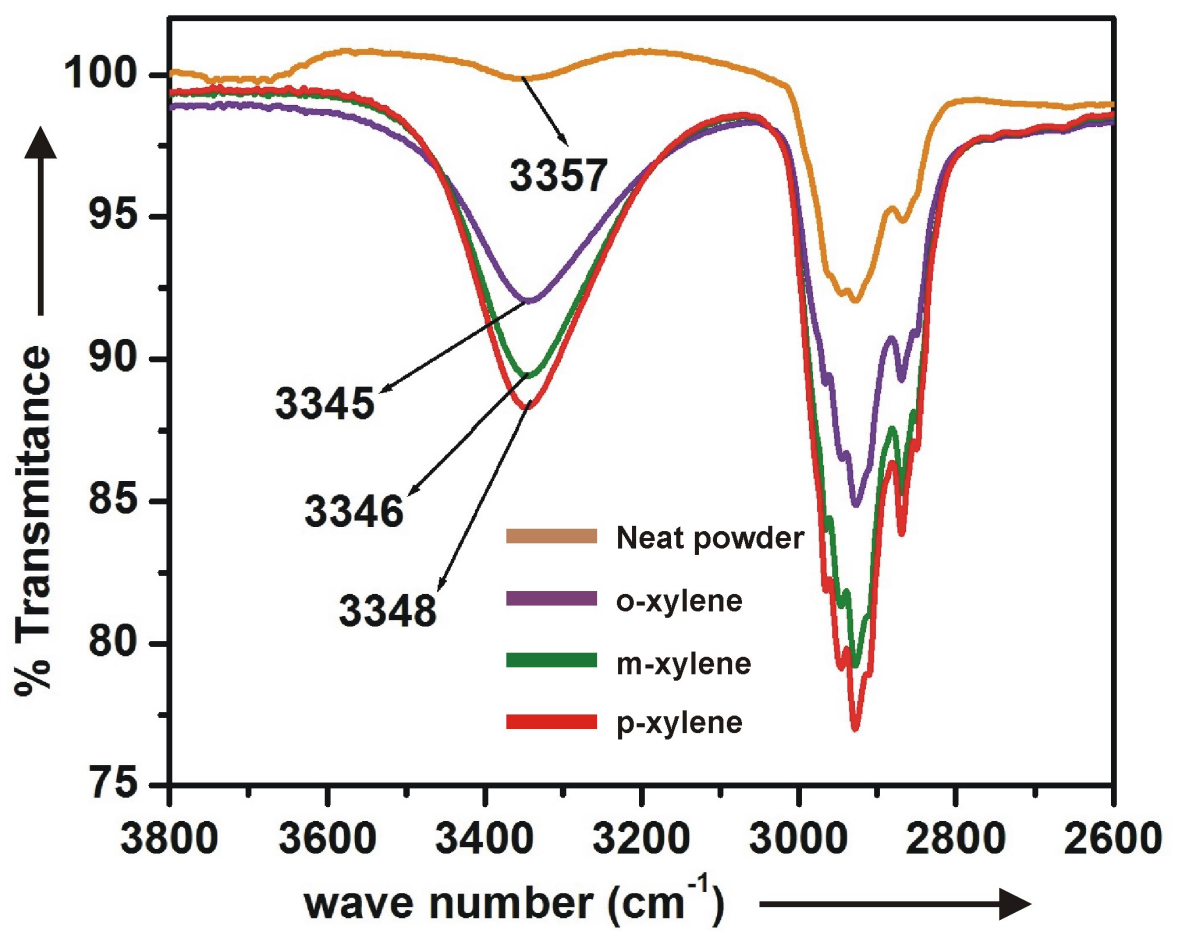

Figure S15: FTIR of neat erythrodiol powder and dried self-assemblies of erythrodiol prepared from o-xylene, m-xylene, p-xylene (1\% w/v), 2-propanol (2\% w/v).

\section{X-ray Diffraction studies:}

\begin{tabular}{|c|c|}
\hline Sample & $\mathrm{d}(\mathrm{nm})$ \\
\hline Neat powder & $1.57,1.28,1.15,0.78,0.65,0.54,0.42,0.36,0.28$ \\
\hline $\begin{array}{l}\text { Dried self-assemblies of } 1 \\
\text { prepared from o-xylene }\end{array}$ & $\begin{array}{l}1.57,1.26,1.15,0.78,0.64,0.53,0.43,0.39,0.35,0.32,0.30 \\
0.29,0.27,0.24\end{array}$ \\
\hline $\begin{array}{l}\text { Dried self-assemblies of } 1 \\
\text { prepared from m-xylene }\end{array}$ & $\begin{array}{l}1.55,1.29,1.09,0.76,0.62,0.52,0.48,0.43,0.32,0.30,0.29 \text {, } \\
0.26,0.23\end{array}$ \\
\hline $\begin{array}{l}\text { Dried self-assemblies of } 1 \\
\text { prepared from } p \text {-xylene }\end{array}$ & $\begin{array}{l}1.57,1.27,1.14,0.77,0.63,0.53,0.44,0.41,0.37,0.34,0.31 \text {, } \\
0.29,0.27,0.26\end{array}$ \\
\hline
\end{tabular}

Table S1: Wide angle X-ray diffraction (XRD) data of neat powder and dried self-assemblies of 1 
(a)

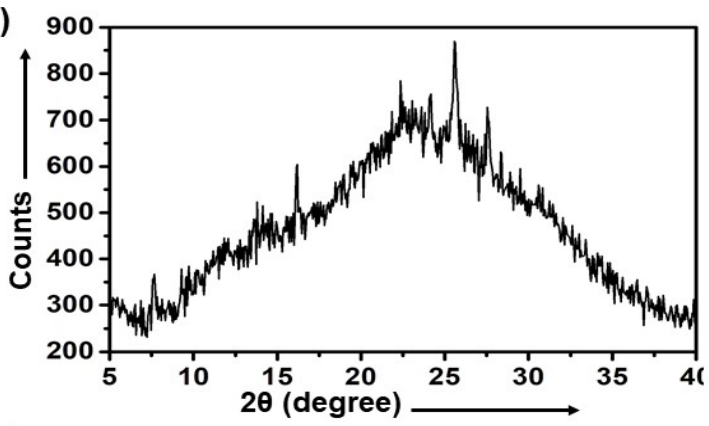

(c)

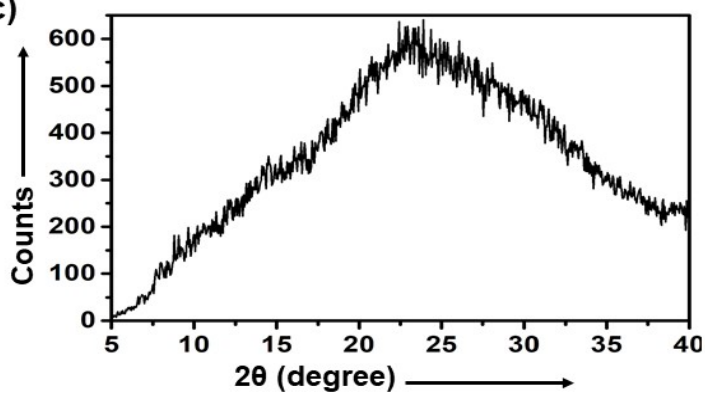

(b)

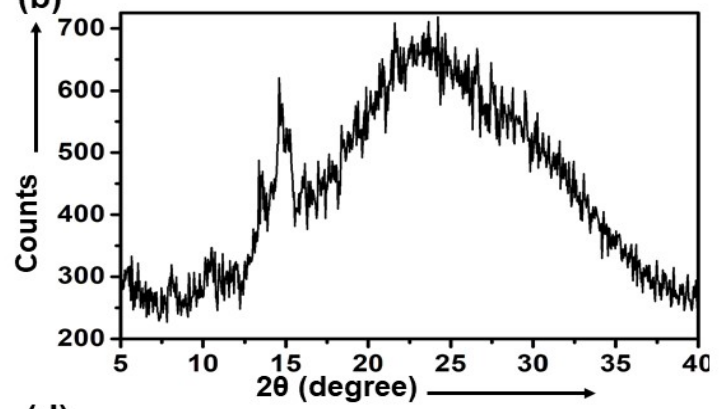

(d)

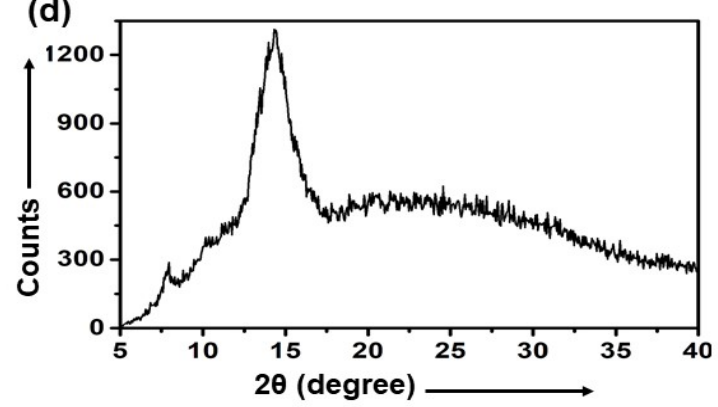

Figure S16: Wide angle powder X-ray diffraction (XRD) data of the dried self-assemblies of 1 prepared from (a) o-xylene $(2 \% \mathrm{w} / \mathrm{v}),(\mathrm{b}) \mathrm{m}$-xylene $(2 \% \mathrm{w} / \mathrm{v}),(\mathrm{c})$ p-xylene $(2 \% \mathrm{w} / \mathrm{v}),(\mathrm{d})$ neat powder of 1

(a)

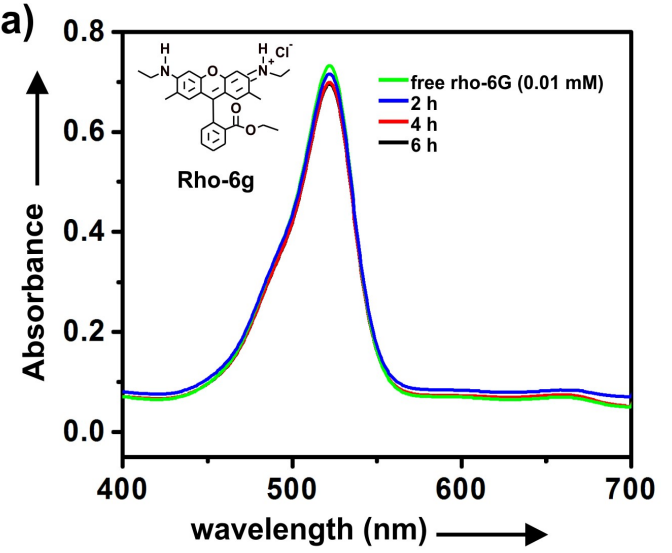

(c)

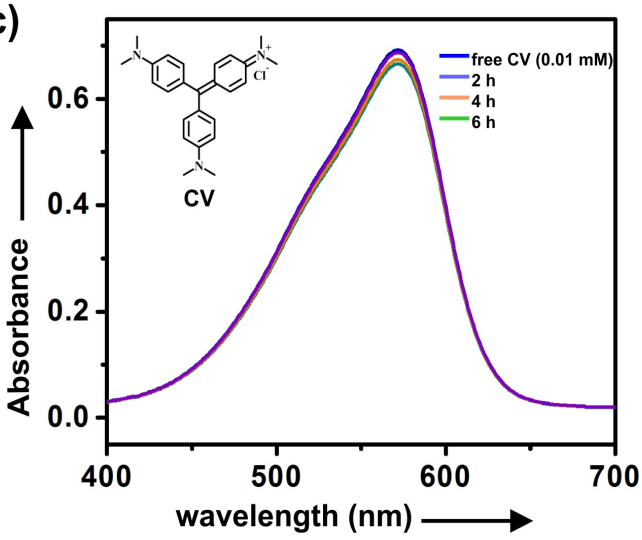

(b)

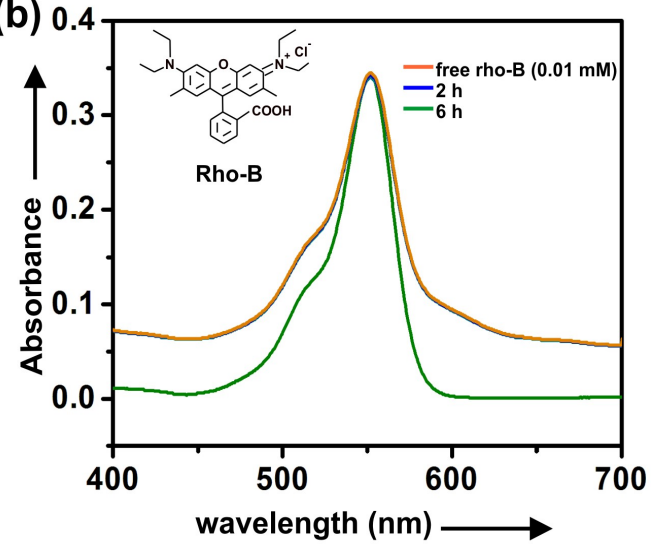

(d)

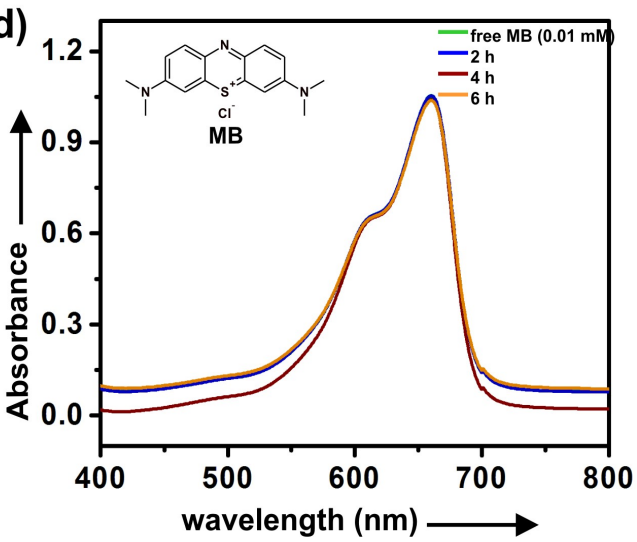

Figure S17: Control experiments for the partition of dyes into odichlorobenzene : (a) Rhodamine B $(0.01 \mathrm{mM})$, (b) Rhodamine $6 \mathrm{G}(0.01$ $\mathrm{mM}),(\mathrm{c})$ Crystal violet $(0.01 \mathrm{mM}),(\mathrm{d})$ methylene blue $(0.01 \mathrm{mM})$. 